\title{
COLLECTIVE SINGULARITIES OF A FAMILY OF ANALYTIC FUNCTIONS ${ }^{1}$
}

GUY JOHNSON, JR.

Let $\mathcal{F}$ denote a family of functions and let $\mathcal{Q}$ denote the radius of the greatest circle with center at $z_{0}$ in which each function is holomorphic and the family is normal. We shall call $R$ the radius of regularity of $\mathcal{F}$ at $z_{0}$. Mandelbrojt has stated recently the following formula for computing $R$ in the case where $\mathcal{F}$ is subuniformly bounded in $\left|z-z_{0}\right|<R$ (i.e. uniformly bounded on each compact subset of $\left.\left|z-z_{0}\right|<R\right)$.

$$
\lim \sup \sup _{f \in \mathscr{F}}\left|a_{n}(f)\right|^{1 / n}=\frac{1}{\mathbb{R}},
$$

where $f(z)=\sum_{n=0}^{\infty} a_{n}(f)\left(z-z_{0}\right)^{n}$.

It may be observed that the formula is in form the same as the Hadamard formula for the radius of convergence of a Taylor series. From the definition of $R$, however, one must consider here the presence of irregular points of a family of functions as well as singular points of a function. In two notes [4] Mandelbrojt presented a composition theorem in which irregular points play a part analogous to that of the singular points in the composition theorem of Hadamard [3]. Montel [6] calls an irregular point of a family a collective singular point. The theorem presented in this paper demonstrates further the analogy between singular point and collective singular point.

$D$ will be called a domain of regularity of $\mathcal{F}$ if each function is holomorphic and the family is normal in $D$. A point $z^{\prime}$ on the boundary of $D$ will be called a regular point of $F$ if there is a neighborhood $\left|z-z^{\prime}\right|<\rho$ such that the union of the neighborhood and $D$ is a domain of regularity. Otherwise $z^{\prime}$ will be called a singular point of $\mathcal{F}$. The theorem is a generalization of the gap theorem of Hadamard [2]. The method of proof is due to Faber [1] and Mordell [5].

THEOREM 1. Let $\varsubsetneqq$ be a family of functions $f(z)=\sum_{n=0}^{\infty} a_{n}(f) z^{n}$ which has radius of regularity $R(\mathcal{F})=1$ and is subuniformly bounded in $|z|<1$. Suppose that to each function there corresponds a sequence of integers $\left\{\lambda_{p}(f)\right\}_{p=1}^{\infty}$ such that $a_{n}(f)=0$ if $n \neq \lambda_{p}(f), p=1,2, \cdots$. More1955.

Presented to the Society, September 3, 1954; received by the editors September $30^{\circ}$

${ }^{1}$ This paper is a portion of a thesis submitted to the faculty of the Rice Institute for the Degree of Doctor of Philosophy. I wish to acknowledge the guidance and inspiration of Professor S. Mandelbrojt during the research here reported. 
over suppose $\lambda_{p+1}(f) / \lambda_{p}(f) \geqq \lambda>1$, where $\lambda$ is independent of $f$. Then each point of $|z|=1$ is a singular point of $F$.

Proof. Choose an integer $q$ such that $q>1 /(\lambda-1)$ and let

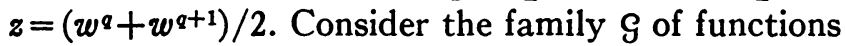

(1) $g(w)=f\left(\frac{w^{q}+w^{q+1}}{2}\right)=\sum_{n=0}^{\infty} a_{n}(f)\left(\frac{w^{q}+w^{q+1}}{2}\right)^{n}=\sum_{m=0}^{\infty} b_{m}(g) w^{m}$.

Now $|w| \leqq 1, w \neq 1$ implies $|z|<1$ so that $R(\mathcal{G}) \geqq 1$ and each point of $|w|=1$ is a regular point of $\mathcal{G}$ except perhaps $w=1$. It will be shown that $R(\mathcal{G})=1$ implying $w=1$ is a singular point of $\mathcal{G}$ and hence $z=1$ is a singular point of $\mathcal{F}$. The family of functions $\sum_{n=0}^{\infty} a_{n}(f) e^{i n \theta} z^{n}$ also satisfies the hypothesis so that $z=e^{i \theta}$ is a singular point of $\mathcal{F}$.

It will be sufficient to show that $R(\mathcal{G}) \leqq 1$. The inequality of the hypothesis together with the choice of $q$ result in the fact that no two polynomials

$$
\left(\frac{w^{q}+w^{q+1}}{2}\right) \lambda_{l}(f) \text { and }\left(\frac{w^{q}+w^{q+1}}{2}\right) \lambda_{k}(f), \quad l \neq k,
$$

have common powers of $w$. Thus each coefficient $b_{m}(g)$ may be expressed in terms of a single coefficient $a_{n}(f)$; specifically

$$
b_{q n+j}(g)=\frac{a_{n}(f)}{2^{n}} C_{j, n}, \quad 0 \leqq j \leqq n .
$$

In particular setting $m_{n}=q n+[n / 2]$

$$
b_{m_{n}}(g)=\frac{a_{n}(f)}{2^{n}} C_{[n / 2], n}
$$

where $[n / 2]$ denotes the integral part of $n / 2$. Then using the fact that

$$
\lim _{n \rightarrow \infty}\left(\frac{C_{[n / 2], n}}{2^{n}}\right)^{1 / m_{n}}=1
$$

and from $R(\mathcal{F})=1$ that $\lim \sup _{f} \sup _{f}\left|a_{n}(f)\right|^{1 / n}=1$, we have

$$
\lim \sup \sup _{n \in \mathcal{G}}\left|b_{m_{n}}\right|^{1 / m_{n}}=\limsup _{n \rightarrow \infty} \sup _{f \in \mathcal{F}}\left|a_{n}(f) \frac{C_{[n / 2], n}}{2^{n}}\right|^{1 / m_{n}}=1 .
$$

Hence

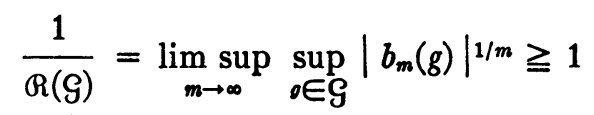

which was to be proved. 


\section{REFERENCES}

1. G. Faber, Über die Nicht-Fortsetzbarkeit gewisser Potenzreihen, Sitz. der Akad. der Wiss. zu München vol. 34 (1904) pp. 63-74.

2. M. J. Hadamard, Essai sur l'étude des fonctions données par leur développement de Taylor, J. Math. Pures et Appl. (4) vol. 8 (1892) p. 116.

3. - Theoreme sur les series entières, Acta Math. vol. 22 (1899) p. 55.

4. S. Mandelbrojt, Sur la composition des familles normales, C. R. Acad. Sci. Paris vol. 186 (1928) pp. 1418 and 1592.

5. L. J. Mordell, On a power series with the circle of convergence as a line of essential singularities, J. London Math. Soc. vol. 2 (1927) pp. 146-148.

6. P. Montel, Leçons sur les fonctions entières ou meromorphes, Paris, GauthierVillars, 1932, p. XI.

Rice INSTITUTE 